\title{
Absorption of Hydrogen in High-strength Low-alloy Steel during Tensile Deformation in Gaseous Hydrogen
}

\author{
Koichi TAKASAWA, Ryoji ISHIGAKI, Yoru WADA and Rinzo KAYANO \\ Muroran Research Laboratory, The Japan Steel Works, Ltd., Muroran 051-8505 Japan.
}

(Received on March 1, 2010; accepted on June 9, 2010)

\begin{abstract}
Absorption of hydrogen in a high-strength nickel-chromium-molybdenum steel during tensile deformation in $0.5 \mathrm{MPa}$ gaseous hydrogen was examined using a thermal desorption analysis method. The tensile strength of the specimen was varied in the range from 1214 to $947 \mathrm{MPa}$ by heat treatment. The dislocation density of the specimens was measured by X-ray diffractometry after tensile testing in a hydrogen atmosphere. The hydrogen content absorbed during tensile deformation increased with increasing tensile strain in proportional elastic range until just before yielding. The yield stress was defined as $0.2 \%$ proof stress in this work. At the same tensile strains, the hydrogen content of lower-strength specimens was larger than that of higher-strength specimens. The dislocation density gradually decreased until just before yielding, corresponding to the proportional increase of hydrogen content to the tensile strain. This implies that the hydrogen absorption behavior during tensile deformation in gaseous hydrogen is related to the motion of mobile dislocations initially contained in the specimens. The activation energy for desorption of hydrogen absorbed during tensile deformation did not depend on the strength of the steel. This indicates that the trap sites of hydrogen atoms created through the tensile deformation were the same regardless of the strength levels.
\end{abstract}

KEY WORDS: hydrogen environment embrittlement; high-strength low-alloy steel; nickel-chromium-molybdenum steel; tensile deformation; hydrogen absorption; diffusive hydrogen; thermal desorption analysis; dislocation; dislocation density; activation energy; trap site; vacancy.

\section{Introduction}

Hydrogen environment embrittlement (HEE) in metals and alloys has been defined as the degradation of mechanical properties such as ductility during deformation in gaseous hydrogen. ${ }^{1)}$ In low-alloy steels, it is well known that the susceptibility of HEE increases as the strength increases. $^{2)}$ Recently, some studies on HEE of carbon steels and high-strength low-alloy steels, which are used for highpressure hydrogen components, ${ }^{3-6)}$ in a high-pressure hydrogen environment have been conducted to evaluate their mechanical properties in this circumstance.

However, the mechanism of HEE has not yet been sufficiently elucidated for those steels. In HEE, the deformation and hydrogen absorption from the environment occur simultaneously. This is considered to make the mechanism of HEE difficult to understand. In other words, a close relationship between the deformation and hydrogen absorption is only inferred. One of the factors affecting HEE is hydrogen transportation by dislocations. Experimental assessments of hydrogen transportation by dislocations have been done for decades. Donovan ${ }^{7)}$ showed that the release rate of tritium from iron and several other steels was accelerated during plastic deformation. Hwang and Bernstein ${ }^{8)}$ demonstrated that edge and screw dislocations have different effects for hydrogen transportation. They also pointed out that when the dislocation velocity reaches a certain value, which is termed break-away velocity, the hydrogen cannot keep up with the dislocation movement. Tabata and Birnbaum $^{9)}$ directly observed the dislocation motion during deformation in gaseous hydrogen in a transmission electron microscope equipped with specially designed environment cells. Their results have been considered one of the experimental proofs that hydrogen makes dislocation mobility increased. Birnbaum ${ }^{10)}$ also concluded that hydrogen transportation by dislocations is a general phenomenon observed in various metals and alloys, and does not depend on the dislocation characteristics; edge, screw and mixed dislocations. Meanwhile, Beachem ${ }^{11)}$ primarily focused on the interaction between dislocations and solute hydrogen, and proposed the theory termed hydrogen-assisted cracking (HAC). Recently, Nagumo et al. ${ }^{12,13)}$ investigated the vacancy formation and aggregation enhanced by hydrogen, and developed a new model on hydrogen embrittlement, "hydrogen-enhanced strain-induced vacancy (HESIV)". Lattice defects formation caused by the annihilation of dislocations with opposite signs was investigated by Essmann and Mughrabi. ${ }^{14)}$

As above described, hydrogen transportation by dislocations is considered an important fundamental process for hydrogen embrittlement. It should be also noted that there are many dislocations in high-strength steels with microstructures of martensite and bainite, and some of them are mobile ones which can move from the early stage 
of deformation. A very low elastic proportional limit observed in a martensitic steel was interpreted to be caused by the movement of those mobile dislocations. ${ }^{15,16)}$ Therefore, in the case of HEE of a high-strength steel, the behavior of mobile dislocations is considered to be related to the hydrogen transportation. In addition, high dislocation density is one of the factors which control the strength of a high-strength steel. It is possible that the relationship between the behavior of mobile dislocations and hydrogen absorption can provide information about the strength dependence of the susceptibility of HEE.

The objective of this study was to evaluate the hydrogen absorption behavior in a high-strength low-alloy steel having several microstructures exhibiting various strength levels during tensile deformation in gaseous hydrogen, especially by examining the change of dislocation density during deformation.

\section{Experimental Procedure}

\subsection{Material and Specimens}

The material used was a commercial nickel-chromiummolybdenum steel, which is specified as Japan Industrial Standard (JIS) G 4053 (2003), SNCM439 steel. The material was a forged plate of $2400 \mathrm{~mm}$ in length, $1000 \mathrm{~mm}$ in width and $75 \mathrm{~mm}$ in thickness. The chemical composition of the material is shown in Table 1. Specimens taken from the material were quenched into the water and tempered under various conditions to achieve the various strength ranging from 947 to $1214 \mathrm{MPa}$. The specimens were termed S1 to S4 in decreasing order of their strength. Heat treatment conditions and tensile strength of the specimens were summarized in Table 2. After heat treatment, platetype tensile test coupons of $19 \mathrm{~mm}$ in overall length (parallel part: $8 \mathrm{~mm}$ ), $4 \mathrm{~mm}$ in width (parallel part: $3 \mathrm{~mm}$ ) and $1 \mathrm{~mm}$ in thickness were taken from the materials by electrical discharge machining. The shape and dimensions of the coupons are shown in Fig. 1. To eliminate the contaminated surface layers formed during the electrical discharge machining, the surfaces of all the coupons were polished by \#800 abrasive paper in the longitudinal direction. All coupons were cleaned in acetone for a few minutes using an ultrasonic cleaner, and then were weighed by analytical balance.

\subsection{Tensile Test in Gaseous Hydrogen and Thermal Desorption Analysis}

As the amount of hydrogen absorbed during deformation was expected to be very small, it was not expected that the absorbed hydrogen was fully discriminated from the initially contained hydrogen. Therefore, prior to the tensile test, the coupons were heated to $873 \mathrm{~K}$ in a quadrupole mass spectrometer (QMS) to remove the hydrogen that was initially contained in the coupons. Then, the hydrogen content of the coupons was measured again to confirm that the initially contained hydrogen was desorbed.

The tensile tests were carried out in a $0.5 \mathrm{MPa}$ pure hydrogen gas environment at room temperature. A specially designed miniature tensile test apparatus that had a load capacity of $5 \mathrm{kN}$ was employed in this study. The crosshead displacement of the apparatus was calibrated and converted
Table 1. Chemical composition of a material used (mass $\%$ ).

\begin{tabular}{|c|c|c|c|c|}
\hline $\mathrm{C}$ & $\mathrm{Si}$ & $\mathrm{Mn}$ & $\mathrm{P}$ & $\mathrm{S}$ \\
\hline 0.42 & 0.22 & 0.80 & 0.014 & 0.0023 \\
\hline $\mathrm{Ni}$ & $\mathrm{Cr}$ & $\mathrm{Mo}$ & $\mathrm{Cu}$ & \multirow{2}{*}{} \\
\cline { 1 - 4 } 1.82 & 0.86 & 0.25 & 0.09 & \\
\hline
\end{tabular}

Table 2. Heat treatment conditions and tensile strength (T.S.).

\begin{tabular}{|c|c|c|c|c|c|c|c|}
\hline \multirow{2}{*}{ Sample } & \multicolumn{3}{|c|}{ Quenching } & \multicolumn{3}{|c|}{ Tempering } & \multirow{2}{*}{$\begin{array}{c}\text { T.S. in air, } \\
\sigma_{\text {UTS }} \\
\text { / MPa }\end{array}$} \\
\hline & $\begin{array}{c}\text { Temp., } \\
T / \mathrm{K}\end{array}$ & $\begin{array}{l}\text { Time, } \\
t / \mathrm{ks}\end{array}$ & Cooling & $\begin{array}{c}\text { Temp., } \\
T / \mathrm{K}\end{array}$ & $\begin{array}{l}\text { Time, } \\
t / \mathrm{ks}\end{array}$ & Cooling & \\
\hline S1 & 1123 & 7.2 & W.Q. & - & - & - & 1214 \\
\hline S2 & 1123 & 7.2 & O.Q. & 853 & 14.4 & A.C. & 1167 \\
\hline \multirow{2}{*}{ S3 } & \multirow{2}{*}{1123} & \multirow{2}{*}{7.2} & \multirow{2}{*}{ O.Q. } & $(1 \mathrm{st}) 853$ & (1st)14.4 & \multirow{2}{*}{ A.C. } & \multirow{2}{*}{977} \\
\hline & & & & (2nd) 893 & (2nd)7.2 & & \\
\hline \multirow{2}{*}{ S4 } & \multirow{2}{*}{1123} & \multirow{2}{*}{7.2} & \multirow{2}{*}{ O.Q. } & $(1 \mathrm{st}) 853$ & (1st)14.4 & \multirow{2}{*}{ A.C. } & \multirow{2}{*}{947} \\
\hline & & & & (2nd)893 & $(2 \mathrm{nd}) 32.4$ & & \\
\hline
\end{tabular}

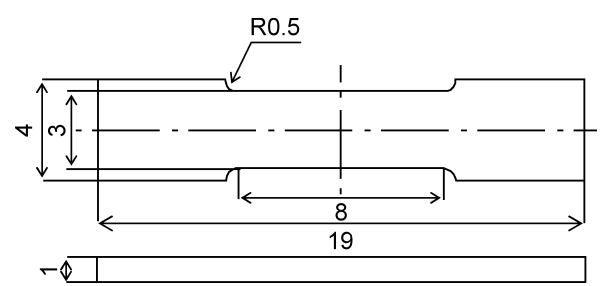

Fig. 1. Shape and dimensions of tensile test coupon ( $\mathrm{mm})$.

to tensile strain using a strain gauge attached to the parallel part of the coupons. The strain rate was set to $1.1 \times 10^{-5} \mathrm{~s}^{-1}$. After setting the coupon in the chamber of the apparatus, the chamber was evacuated to a pressure below $10^{-3} \mathrm{~Pa}$. Next, the chamber was purged by hydrogen gas, which purity was higher than $99.99999 \%$, to remove the moisture and other remained gaseous substances. The purging consists of the following procedures: firstly, $0.5 \mathrm{MPa}$ hydrogen was introduced into the chamber, and then the hydrogen was released immediately to atmospheric pressure. The purging was repeated for five times, and $0.5 \mathrm{MPa}$ hydrogen was finally introduced into the chamber for the tensile test. By purging, the oxygen content of the hydrogen gas in the chamber was suppressed below 1 ppm.

Because of the high diffusivity of hydrogen in ferritic steels, rapid handling was required after the tensile test to correctly measure the hydrogen content. This tensile test apparatus was equipped with a dedicated vessel for transporting the coupon from the apparatus to QMS without exposure to air. Prior to the tensile test, the atmosphere in the vessel was preliminarily substituted with dry nitrogen. After the tensile test in hydrogen, the coupon was quickly detached from the gripping device and put into the vessel, and then transported to the QMS. With the use of this vessel, the hydrogen desorption analysis could be started within $10 \mathrm{~min}$ after finishing the tensile test. In the previous work, ${ }^{17)}$ the hydrogen absorbed in a high-strength steel during tensile deformation in $0.5 \mathrm{MPa}$ gaseous hydrogen was confirmed to be remained even if the steel was exposed to air in room temperature for several hours. 
Thermal desorption analysis (TDA) was performed using the QMS in the temperature range from room temperature to $873 \mathrm{~K}$. The heating rate was varied between $200 \mathrm{~K} \cdot \mathrm{h}^{-1}$ and $1800 \mathrm{~K} \cdot \mathrm{h}^{-1}$. The activation energy for hydrogen desorption, $E_{\mathrm{a}}$, was calculated by the following equation ${ }^{18)}$ :

$$
\frac{E_{\mathrm{a}} \phi}{R T_{\mathrm{p}}^{2}}=A \exp \left(-\frac{E_{\mathrm{a}}}{R T_{\mathrm{p}}}\right)
$$

where $\phi, R, T_{\mathrm{p}}$ and $A$ are the heating rate, gas constant, peak temperature in the desorption profile and constant, respectively. A slope of the straight line, which is obtained by plotting the logarithm of $\phi / T_{\mathrm{p}}^{2}$ against $T_{\mathrm{p}}{ }^{-1}$, gives $E_{\mathrm{a}} / R$.

\subsection{Dislocation Density Measurement}

The dislocation density, $\rho$, of the parallel portion of the coupons after tensile deformation in hydrogen was determined using X-ray diffractometry with the following procedures: firstly, the local strain, $\varepsilon_{1}$, was estimated by Hall's method $^{19)}$ as shown in Eq. (2):

$$
\beta_{\mathrm{t}}\left(\frac{\cos \theta}{\lambda}\right)=\frac{K}{D}+2 \varepsilon_{1}\left(\frac{\sin \theta}{\lambda}\right)
$$

where $\theta, \lambda, K$ and $D$ are the diffraction angle, wavelength of the X-ray, Scherrer constant (0.89), and crystallite size, respectively. A slope of the straight line, which is obtained by plotting the left term of Eq. (2) against $(\sin \theta) / \lambda$, gives $\varepsilon_{l} . \beta_{\mathrm{t}}$ is the true Full Width at the Half Maximum height (FWHM), which is derived from Eq. (3), where $\beta_{\mathrm{s}}$ and $\beta_{0}$ are the FWHM of each coupon and pure iron, respectively:

$$
\beta_{\mathrm{t}}^{2}=\beta_{\mathrm{s}}^{2}-\beta_{0}^{2}
$$

Next, Eq. (4) was employed to estimate the dislocation density $^{20)}$ :

$$
\rho=\frac{14.4 \times \varepsilon_{1}^{2}}{\mathbf{b}}
$$

where $\mathbf{b}$ is the Burgers vector set to $0.25 \mathrm{~nm}$ in this work.

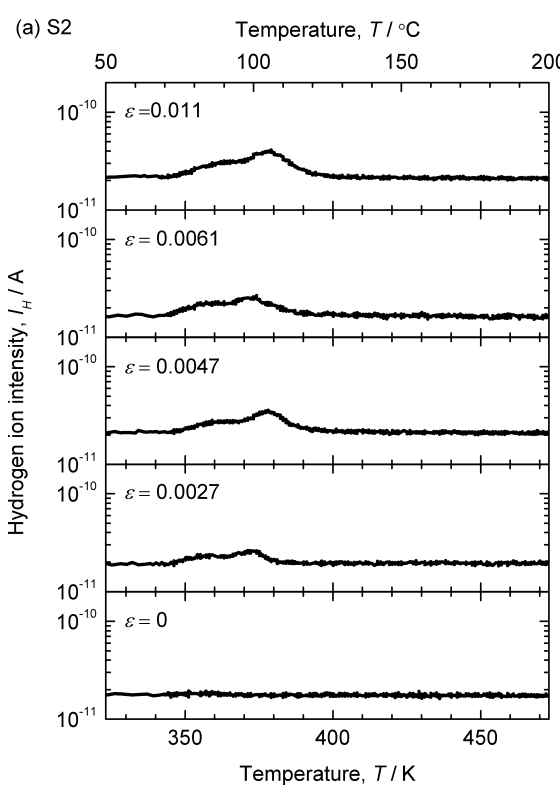

\section{Results}

\subsection{Hydrogen Content Absorbed in the Steel During Tensile Deformation in Hydrogen}

Figures 2(a) and 2(b) show the hydrogen thermal desorption profiles at various tensile strains, $\varepsilon$, of $\mathrm{S} 2$ and $\mathrm{S} 3$, respectively. Every profile was measured at the heating rate of $200 \mathrm{~K} \cdot \mathrm{h}^{-1}$. The condition of $\varepsilon=0$ means that the coupon was not deformed, but exposed to $0.5 \mathrm{MPa}$ hydrogen for $30 \mathrm{~min}$ at room temperature. No obvious peaks were observed in both profiles of $\varepsilon=0$, indicating that hydrogen does not penetrate the coupons only by hydrogen exposure. As the coupon began to deform, peaks emerged at approximately 340 to $400 \mathrm{~K}$. The peaks became more pronounced with increasing tensile strain. Because the peaks appeared only in the coupons subject to tensile deformation in a hydrogen atmosphere, it is considered that these are attributable to the hydrogen that absorbed during tensile deformation.

The tensile strain dependence on hydrogen content is shown in Fig. 3 as well as the typical stress-strain curves, where $C_{\mathrm{H}}$ represents the content of hydrogen desorbed at the temperatures below $423 \mathrm{~K}$ in the profiles of Fig. 2 . The yield stress was defined as $0.2 \%$ proof stress in this work, and all of the $C_{\mathrm{H}}$ except that of S1 and S2 in $\varepsilon>0.006$ were within the proportional limit. $C_{\mathrm{H}}{ }^{*}$ represents the hydrogen content of the coupon deformed up to a designated tensile strain in a $0.1 \mathrm{MPa}$ nitrogen, and subsequently exposed to $0.5 \mathrm{MPa}$ hydrogen for the same time as that for tensile deformation in nitrogen with keeping the strain. $C_{\mathrm{H}}{ }^{*}$ also represents the content of hydrogen desorbed at temperatures below $423 \mathrm{~K}$. The difference of strength was distinctly reflected to the hydrogen content. $C_{\mathrm{H}}$ in $\mathrm{S} 1$ and $\mathrm{S} 2$ was almost the same with each other and that in S3 and S4 was so. However $C_{\mathrm{H}}$ in $\mathrm{S} 3$ and $\mathrm{S} 4$ was larger than that in $\mathrm{S} 1$ and $\mathrm{S} 2$ at the same tensile strain. In $\mathrm{S} 1$ and $\mathrm{S} 2, C_{\mathrm{H}}$ increased with increased tensile strain up to about 0.005 , and then did not show monotonic increase with tensile strain. Meanwhile, $C_{\mathrm{H}}$ linearly increased with the strain up to about

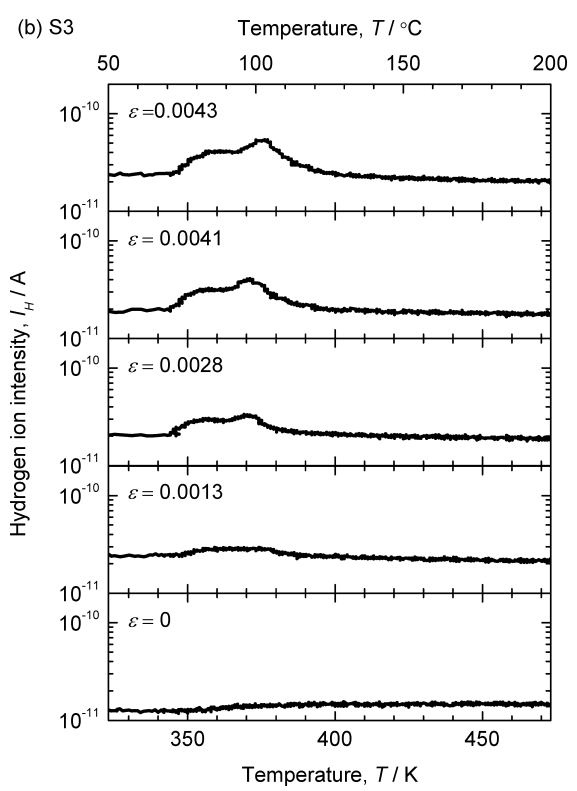

Fig. 2. Temperature dependence of hydrogen desorption at various tensile strains; (a) S2 and (b) S3. The heating rate was $200 \mathrm{~K} \cdot \mathrm{h}^{-1}$. 
0.004 in $\mathrm{S} 3$ and $\mathrm{S} 4 . C_{\mathrm{H}}{ }^{*}$ in all the specimens did not change regardless of the tensile strain.

The change in dislocation density as a function of tensile strain is shown in Fig. 4. The error bars correspond to the deviation of data from the straight line obtained by Eq. (2). The dislocation density of S1 was equivalent to that of S2, and similarly, those of S3 and S4 were almost equal. However, the dislocation density of S1 and S2 was about an order of magnitude larger than that of S3 and S4 in the strain up to $\varepsilon=0.004$. The dislocation density of S1 and S2 at $\varepsilon<0.005$ was almost constant, and then it increased around $\varepsilon=0.0055$. Meanwhile the dislocation density of S3

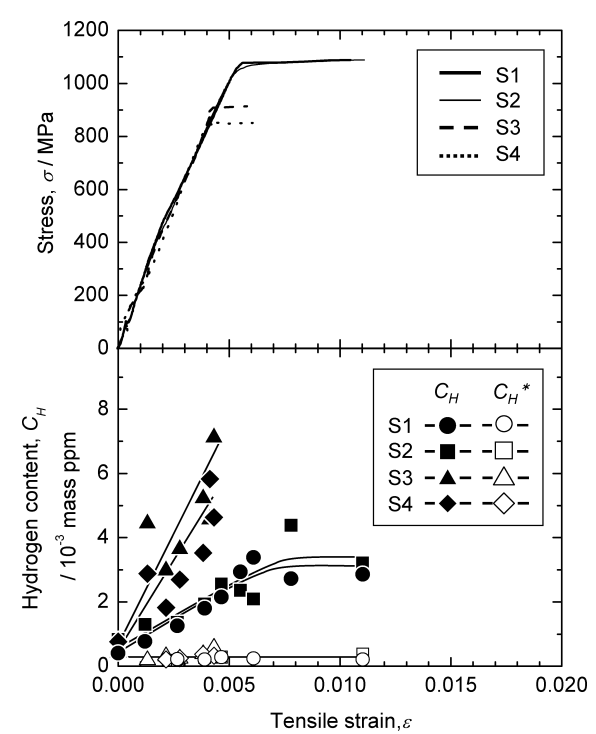

Fig. 3. Relationship between tensile strain and hydrogen content desorbed below $423 \mathrm{~K}$. The upper diagram shows the typical stress-strain curve of specimens. $C_{\mathrm{H}} *$ represents the hydrogen content of the coupon deformed up to a designated tensile strain in a $0.1 \mathrm{MPa}$ nitrogen, and subsequently exposed to $0.5 \mathrm{MPa}$ hydrogen for the same time as that for tensile deformation in nitrogen with keeping the strain.

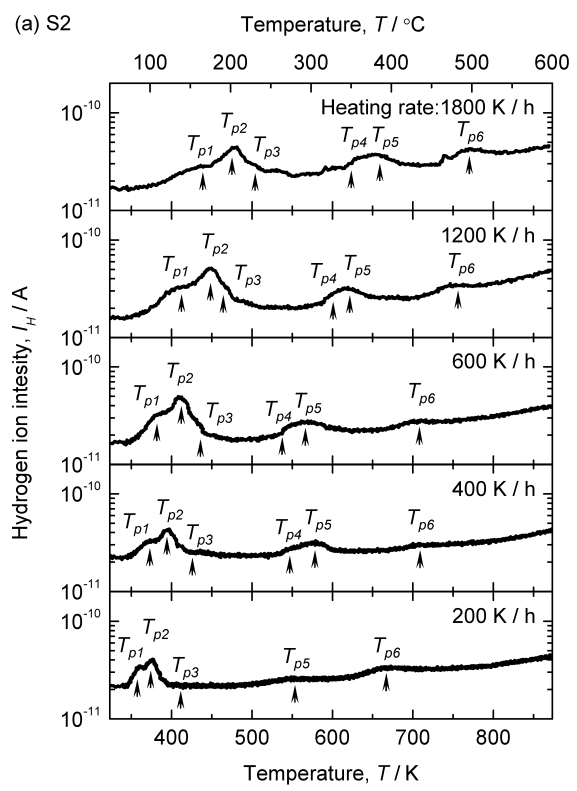

and S4 was also nearly constant at $\varepsilon<0.004$, and that of S4 increased rapidly around $\varepsilon=0.0045$.

\subsection{Activation Energy for Hydrogen Desorption}

Figures 5(a) and 5(b) show the hydrogen desorption behaviors of S2 and S3 at various heating rates, respectively. All coupons were deformed until the stress reached the tensile strength. In every heating rate, some desorption peaks appeared. Because the absorbed hydrogen was very small in this study, hydrogen was considered to be trapped in accordance with Boltzmann distribution. ${ }^{21)}$ So, assuming that the obtained desorption profiles were the combination of the Gaussian curves corresponding to each trap sites, the peak temperatures $T_{\mathrm{p} 1}$ to $T_{\mathrm{p} 6}$ (in order of increasing temperature) were determined. $T_{\mathrm{p} 1}$ to $T_{\mathrm{p} 3}$ correspond to the desorption peaks that appeared after tensile deformation in a 0.5 MPa hydrogen. The peak temperatures shifted to lower ones with decreasing heating rates. Corresponding to the peak temperatures $T_{\mathrm{p} 1}$ to $T_{\mathrm{p} 6}$, the activation energies $E_{\mathrm{a} 1}$ to $E_{\mathrm{a} 6}$ were determined as summarized in Table 3. $E_{\mathrm{a} 1}$ to $E_{\mathrm{a} 3}$ ranged from 20 to $40 \mathrm{~kJ} \cdot \mathrm{mol}^{-1}$, which are not considered a

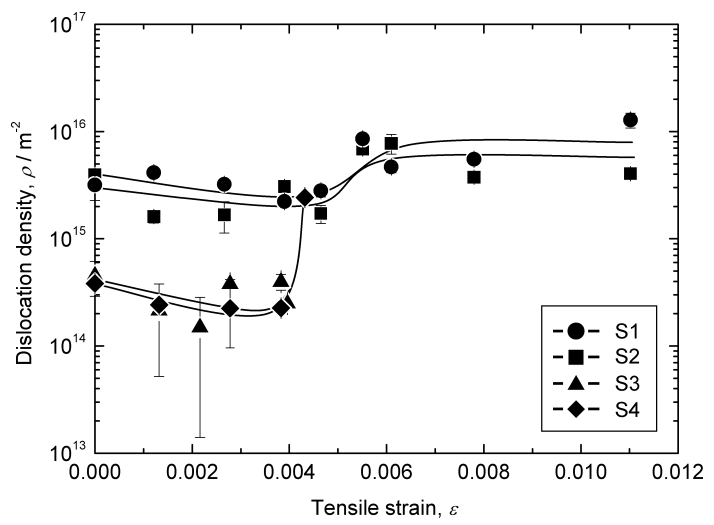

Fig. 4. Tensile strain dependence of dislocation density. Dislocation density was estimated using local strain obtained by Hall's method. ${ }^{19)}$ The error bars correspond to the deviation of data from the straight line obtained by Eq. (2).

(b) $\mathrm{S} 3$

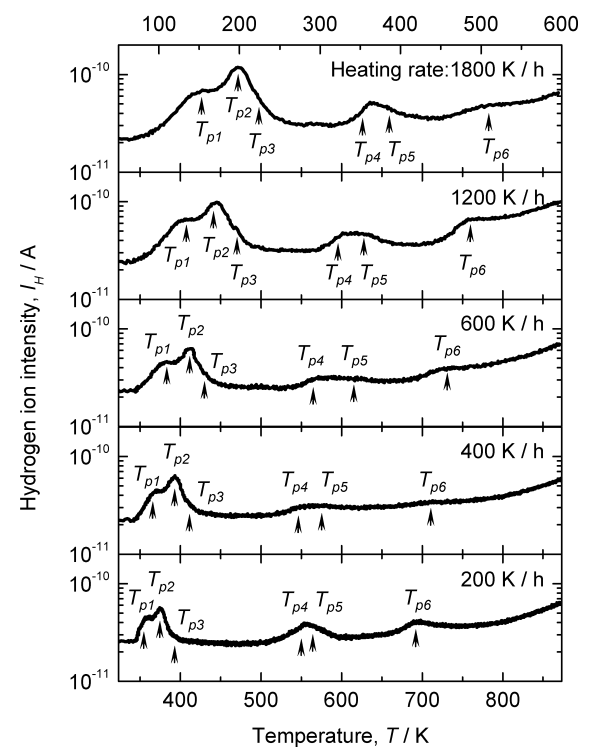

Fig. 5. Temperature dependence of hydrogen desorption at various heating rates; (a) S2 and (b) S3. The coupons of each profile were loaded up to tensile strength. The arrows and designations $T_{\mathrm{p} 1}$ to $T_{\mathrm{p} 6}$ indicate peak temperatures. 
Table 3. Activation energy for hydrogen desorption.

\begin{tabular}{|c|c|c|c|c|c|c|c|}
\hline \multirow{2}{*}{ Sample } & Tensile strength & \multicolumn{5}{|c|}{ Activation energy for hydrogen desorption, $E_{a} / \mathrm{kJ}^{\mathrm{i}} \cdot \mathrm{mol}^{-1}$} \\
\cline { 3 - 8 } & $/ \mathrm{MPa}$ & $\sigma_{\mathrm{U} 1}$ & $E_{\mathrm{a} 2}$ & $E_{\mathrm{a} 3}$ & $E_{a 4}$ & $E_{\mathrm{a} 5}$ & $E_{\mathrm{a} 6}$ \\
\hline S1 & 1214 & $36.7 \pm 1.1$ & $26.5 \pm 0.9$ & $24.6 \pm 0.7$ & $40.6 \pm 2.6$ & $44.5 \pm 3.8$ & $89.0 \pm 13.1$ \\
\hline S2 & 1163 & $27.1 \pm 2.5$ & $24.1 \pm 1.4$ & $20.4 \pm 0.9$ & $33.7 \pm 6.4$ & $45.5 \pm 8.8$ & $75.5 \pm 9.3$ \\
\hline S3 & 977 & $29.2 \pm 2.7$ & $25.3 \pm 1.3$ & $23.4 \pm 1.5$ & $52.7 \pm 8.8$ & $49.7 \pm 7.5$ & $88.6 \pm 13.8$ \\
\hline S4 & 947 & $32.7 \pm 2.6$ & $25.7 \pm 0.8$ & $22.2 \pm 1.0$ & $44.2 \pm 3.7$ & $45.5 \pm 4.9$ & $81.3 \pm 6.8$ \\
\hline
\end{tabular}

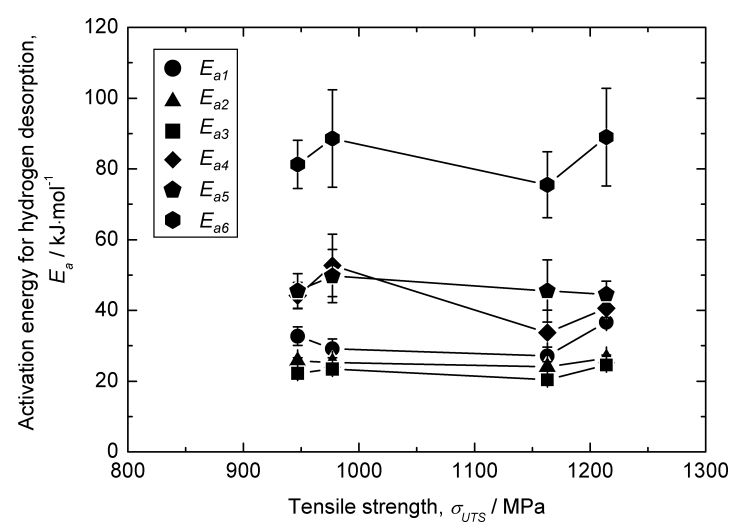

Fig. 6. Relationship between tensile strength and activation energy for hydrogen desorption. $E_{\mathrm{a} 1}$ to $E_{\mathrm{a} 6}$ correspond to the peak temperatures $T_{\mathrm{p} 1}$ to $T_{\mathrm{p} 6}$ in Fig. 6 . The error bars are attributed to the deviation of data from the straight line obtained by Eq. (1).

remarkable difference. $T_{\mathrm{p} 4}$ and $T_{\mathrm{p} 5}$, were located nearly each other, resulting in the comparable activation energies of $E_{\mathrm{a} 4}$ and $E_{\mathrm{a} 5} . E_{\mathrm{a} 6}$ was the largest energy in every specimen.

Figure 6 presents the relationship between tensile strength and activation energies for hydrogen desorption. The error bars correspond to the deviation of data from the straight line obtained by Eq. (1). There was no clear relationship between activation energies and tensile strength.

\section{Discussion}

\subsection{Hydrogen Absorption behavior During Tensile Deformation in Hydrogen Gas}

As indicated by the desorption profiles in Fig. 2, hydrogen absorption began just after the start of tensile deformation. Diffusion may be one of the major hydrogen absorption factors in addition to hydrogen transportation by dislocations. However, if hydrogen absorption is due to diffusion, the hydrogen content would be expected to increase with increasing exposure time to hydrogen. For instance, in the case of $\varepsilon=0.011$ in Fig. 2(a), the deformation process time was about 17 min until the strain reached to this strain $(\varepsilon=0.011)$. Even if the exposure time in the hydrogen before tensile test is taken into account, the total time that the coupon was exposed to $0.5 \mathrm{MPa}$ hydrogen was less than $20 \mathrm{~min}$. On the other hand, in the case of $\varepsilon=0$ in Fig. 2(a), hydrogen absorption was not recognized even after $30 \mathrm{~min}$ of exposure to hydrogen. Therefore, it is difficult to attribute the hydrogen absorption mechanism to diffusion only. In addition, the change in $C_{\mathrm{H}}{ }^{*}$ shown in Fig. 3 does not indicate the contribution of diffusion to the hydrogen absorption; regardless of static strain is applied, hydrogen was hardly absorbed in the specimens from the environ-

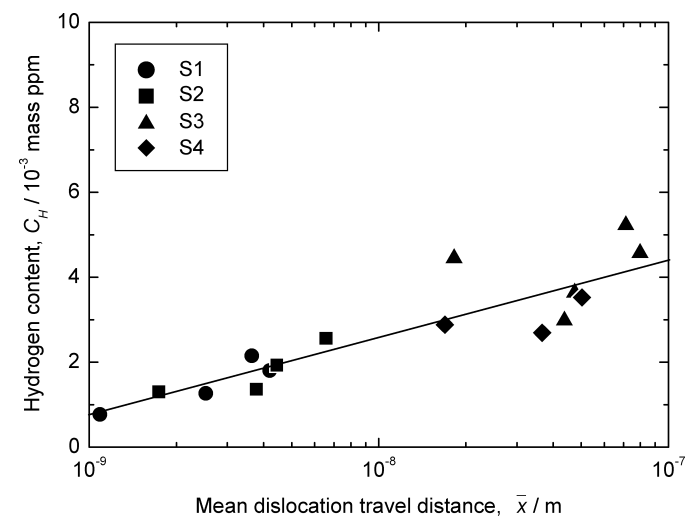

Fig. 7. Relationship between the mean dislocation travel distance and hydrogen content desorbed below $423 \mathrm{~K}$. The data of this figure correspond to the tensile strain region $\varepsilon<0.004-0.005$ in Fig. 3. Mean dislocation travel distance $\bar{x}$ are estimated by $\bar{x}=\varepsilon / \rho \mathbf{b}$, where $\varepsilon, \rho$, and $\mathbf{b}$ are tensile strain, dislocation density and Burgers vector $(0.25 \mathrm{~nm})$, respectively.

ment by diffusion. This result implies that the deformation had a significant effect on the hydrogen absorption behavior from the gaseous hydrogen environment.

It was confirmed that the hydrogen content increase with increasing tensile strain just after beginning of tensile deformation in a high-strength low-alloy steel. ${ }^{17)}$ In this study, a proportional increase of hydrogen content lasted up to approximately $\varepsilon=0.005$ for S1 and S2, and $\varepsilon=0.004$ for S3 and S4. These strains correspond to those for the commencement of macroscopic plastic deformation. It demonstrates that hydrogen was absorbed in the elastic region before the steel macroscopically yielded.

Moreover, this study revealed the clear difference of hydrogen absorption depending on the strength of the steel: more hydrogen was absorbed in the steel having lower strength than that having higher strength. A martensitic steel often has a myriad of dislocations that are introduced at the martensitic transformation, and the dislocation density plays a governing role for the strength. However, the dislocation density of a martensitic steel decreases in the early stage of deformation, meaning the activity of the mobile dislocation follows the mutual annihilation with those with the opposite sign. ${ }^{22}$ Assuming that the local plastic deformation occurred in the macroscopic elastic deformation region, dislocation density was considered to increase locally. The increase of dislocation due to local plastic deformation is offset by the annihilation of mobile dislocations, resulting in constant dislocation density in the early stage of deformation shown in Fig. 4.

The dislocation theory of deformation gives the relationship of dislocation density, $\rho$, strain, $\varepsilon$, and dislocation travel distance, $x$, with the following equation ${ }^{23)}$.

$$
x=\frac{\varepsilon}{\rho \mathbf{b}}
$$

where $\mathbf{b}$ is the Burgers vector. Under an assumption that the value of $\rho$ in Eq. (5) is the average dislocation density between an undeformed specimen and that deformed to a certain tensile strain, the mean dislocation travel distance, $\bar{x}$, during the tensile deformation is estimated. In Fig. 7, the values of $C_{\mathrm{H}}$ in Fig. 3 are plotted against the mean disloca- 
tion travel distance in the early stage of tensile deformation obtained by the above mentioned procedure. Here, the early stage of tensile deformation was taken into account, which corresponded to the tensile strain region $\varepsilon<0.004-0.005$ where the dislocation density was nearly constant in Fig. 4.

A linear relationship is observed between $C_{\mathrm{H}}$ and mean dislocation travel distance, implying that hydrogen absorption in the early stage of tensile deformation is affected by the motion of mobile dislocations, namely by its mean travel distance. In the mechanism of hydrogen embrittlement, plastic deformation plays a significant role. ${ }^{24,25)}$ However, the results of this study demonstrate that the process before macroscopic plastic deformation also influences on HEE in a high-strength steel.

In Fig. 4 the dislocation density increased around $\varepsilon=$ 0.0055 for $\mathrm{S} 1$ and $\mathrm{S} 2$, and around $\varepsilon=0.0045$ for S4. It is considered that screw dislocations mainly govern the process of plastic deformation in body-centered cubic (BCC) metals, and they begin to multiply as yielding begins. Thus, the rapid increase of dislocation density can be interpreted as the multiplication of screw dislocations. However, the dislocation density in S1 and S2 already reached quite a large value on the order of $10^{15} \mathrm{~m}^{-2}$, which is considered the general dislocation density of martensitic steels. ${ }^{26)}$ So, the increase of dislocation density was smaller in S1 and S2 than that in S4. This difficulty of multiplication probably suppressed the absorption of hydrogen in S1 and $\mathrm{S} 2$ at $\varepsilon=0.005$ or more, as shown in Fig. 3.

\subsection{Activation Energy for Hydrogen Desorption}

The existence states of hydrogen, that is, what trap sites capture hydrogen, can be reckoned by evaluating the activation energy for hydrogen desorption. As shown in Fig. 6, six kinds of activation energies are obtained in this study and they did not apparently depend on the strength of the steel, indicating that the same trap sites are present, or generated during tensile deformation in hydrogen regardless of the strength of the steel. The effect of trap sites having small binding energy less than $20 \mathrm{~kJ} \cdot \mathrm{mol}^{-1}$ such as solute carbon and nitrogen ${ }^{27)}$ were not detected in the present work.

Because obvious hydrogen desorption peaks after tensile testing were confirmed, and the content of absorbed hydrogen was related to the dislocation density, it is possible that any of the activation energies of $E_{\mathrm{a} 1}$ to $E_{\mathrm{a} 3}$ were attributable to dislocations. The coupons used for TDA shown in Fig. 5 were provided plastic deformation. So, $E_{\mathrm{a} 1}$ to $E_{\mathrm{a} 3}$ were due to screw dislocations as well as edge dislocations. Some studies have reported that the binding energy between hydrogen and dislocation is on the order of $20 \mathrm{~kJ}$. $\left.\mathrm{mol}^{-1} \cdot 18,28,29\right)$ The activation energy for desorption from a trap site, $E_{\mathrm{a}}$, is the sum of the binding energy between hydrogen and the trap site, $E_{\mathrm{b}}$, and the migration energy, $E_{\mathrm{m}}$. Unless the concrete value of migration energy is known, accurate evaluation of the binding energy between hydrogen and dislocation have not been conducted so far. Gu et al. ${ }^{30)}$ also calculated the binding energy of bainite or martensite lath boundaries as $28.1 \mathrm{~kJ} \cdot \mathrm{mol}^{-1}$. Moreover, because the values of $E_{\mathrm{a} 1}$ to $E_{\mathrm{a} 3}$ were comparable and relatively small, a local-equilibrium state ${ }^{29)}$ is possibly achieved in the trap sites corresponding to $E_{\mathrm{a} 1}$ to $E_{\mathrm{a} 3}$. Therefore, the trap sites that had energies $E_{\mathrm{a} 1}$ to $E_{\mathrm{a} 3}$ could not be individually identified. Accordingly, these trap sites should be treated as a type of low-energy sites, including dislocations.

Dislocation interaction like cutting and mutual annihilation is expected to generate point defects, such as vacancies. Previous studies have presented the binding energy between hydrogen and vacancy, ${ }^{31,32)}$ which is somewhat larger than that of hydrogen and dislocation. Lee et al. ${ }^{33)}$ reported that the activation energy of microvoids was $48.3 \mathrm{~kJ} \cdot \mathrm{mol}^{-1}$ using a similar steel to this work. It is possible that the trap sites of $E_{\mathrm{a} 4}$ or $E_{\mathrm{a} 5}$ corresponded to vacancies and microvoids. However, grain boundaries have also been reported as the trap sites with similar orders of energies. ${ }^{34)}$ Therefore, deliberate evaluations are also required to determine the trap sites related to vacancies. The trap site of the highest energy, $E_{\mathrm{a} 6}$ in this study, seems to be related to non-metallic inclusions ${ }^{33)}$ and precipitations. ${ }^{35)}$

\section{Conclusions}

This work describes the experimental evidence of hydrogen absorption in a high-strength nickel-chromium-molybdenum steel with various strength levels adjusted by heat treatment during tensile deformation in a $0.5 \mathrm{MPa}$ gaseous hydrogen. Obtained results are summarized as follows:

(1) The content of hydrogen absorbed during tensile deformation increased with increasing tensile strain before macroscopic yielding. Compared at the same tensile strains, more hydrogen was absorbed by the steel of lower strength than the steel of higher strength.

(2) The change in hydrogen content was related to the change in dislocation density, which especially implies that the annihilation of mobile dislocations may affect the absorption behavior of hydrogen.

(3) The activation energy for hydrogen desorption did not depend on the strength of the steel. This indicates that the same kinds of trap sites are generated during tensile deformation in a $0.5 \mathrm{MPa}$ gaseous hydrogen regardless of the strength of the steel.

\section{Acknowledgment}

This study was commissioned by the New Energy and Industrial Technology Development Organization (NEDO), Japan, an independent administrative agency.

\section{REFERENCES}

1) H. R. Gray: ASTM STP, 543 (1974), 133.

2) W. T. Chandler and R. J. Walter: ASTM STP, 543 (1974), 170.

3) K. Takasawa, Y. Wada, R. Ishigaki and R. Kayano: Mater. Trans., 51 (2010), 347

4) S. Matsuoka, N. Homma, H. Tanaka, Y. Fukushima and Y. Murakami: J. Jpn. Inst. Met., 70 (2006), 1002.

5) M. Imade, S. Fukuyama, L. Zhang, M. Wen and K. Yokogawa: J. Jpn. Inst. Met., 69 (2005), 190.

6) L. Zhang, M. Wen, S. Fukuyama and K. Yokogawa: J. Jpn. Inst. Met., 67 (2003), 460.

7) J. A. Donovan: Metall. Trans. A, 7 (1976), 1677.

8) C. Hwang and I. M. Bernstein: Acta Metall., 34 (1986), 1001

9) T. Tabata and H. K. Birnbaum: Scr. Metall., 18 (1984), 231.

10) H. K. Birnbaum: Proc. of 4th Int. Conf. on the Effect of Hydrogen on the Behavior of Materials, TMS, Warrendale, PA, (1990), 639.

11) C. D. Beachem: Metall. Trans., 3 (1972), 437. 
ISIJ International, Vol. 50 (2010), No. 10

12) M. Nagumo: Mater. Sci. Technol., 20 (2004), 940.

13) M. Nagumo, M. Nakamura and K. Takai: Metall. Mater. Trans. A, 32 (2001), 339

14) U. Essmann and H. Mughrabi: Philos. Mag. A, 40 (1979), 731.

15) K. Sugimoto, T. Sakaki, T. Fukusato and O. Miyagawa: Tetsu-toHagané, 70 (1984), 1712.

16) T. Sakaki, K. Sugimoto and O. Miyagawa: Tetsu-to-Hagané, 67 (1981), 2172

17) K. Takasawa, Y. Wada, R. Ishigaki and Y. Tanaka: Proc. of 2008 Int Hydrogen Conf., ASM Int., Materials Park, OH, (2009), 532.

18) W. Y. Choo and J. Y. Lee: Metall. Trans. A, 13 (1982), 135.

19) W. H. Hall: Proc. Phys. Soc. A, 62 (1949), 741.

20) G. K. Williamson and R. E. Smallman: Philos. Mag., 8 (1956), 34

21) M. Nagumo: Zairyo-to-Kankyo, 54 (2005), 306.

22) N. Takamura: Machine Design, 48 (2004), 130.

23) M. Kato: Introduction to the Theory of Dislocations, Shokabo,
Tokyo, (1999), 12.

24) M. Nagumo: Bull. Jpn. Inst. Met., 21 (1982), 672.

25) M. Nagumo and K. Miyamoto: J. Jpn. Inst. Met., 45 (1981), 1309.

26) S. Takaki: J. Jpn. Inst. Light Met., 56 (2006), 609.

27) J. J. Au and H. K. Birnbaum: Acta Metall., 26 (1978), 1105.

28) H. Hagi and Y. Hayashi: J. Jpn. Inst. Met., 49 (1985), 327.

29) R. A. Oriani: Acta Metall., 18 (1970), 142.

30) J. L. Gu, K. D. Chang, H. S. Fang and B. Z. Bai: ISIJ Int., 42 (2002), 1560.

31) S. M. Myers, D. M. Follstaedt, F. Besenbacher and J. Bøttiger: J. Appl. Phys., 53 (1982), 8734

32) S. M. Myers, S. T. Picraux and R. E. Stoltz: J. Appl. Phys., 50 (1979), 5710.

33) J. L. Lee and J. Y. Lee: Met. Sci., 17 (1983), 426.

34) K. Ono and M. Meshii: Acta Metall. Mater, 40 (1992), 1357.

35) J. P. Hirth: Metall. Trans. A, 11 (1980), 861. 